\title{
Impact of Different Embolic Agents for Transarterial Chemoembolization (TACE) Procedures on Systemic Vascular Endothelial Growth Factor (VEGF) Levels
}

\author{
Andreas Schicho* ${ }^{1}$, Claus Hellerbrand ${ }^{2}$, Kristina Krüger ${ }^{3}$, Lukas P. Beyer ${ }^{1}$, \\ Walter Wohlgemuth ${ }^{1}$, Christoph Niessen ${ }^{1}$, Ernst Hohenstein ${ }^{3}$, Christian Stroszczynski ${ }^{1}$, \\ Philippe L. Pereira ${ }^{3}$ and Philipp Wiggermann ${ }^{1}$ \\ ${ }^{1}$ Department of Radiology, University Hospital Regensburg, Regensburg, Germany; ${ }^{2}$ Friedrich-Alexander University Erlangen- \\ Nürnberg, Institute of Biochemistry (Emil-Fischer Zentrum), Erlangen, Germany; ${ }^{3}$ Clinic for Radiology, Minimally-invasive \\ Therapies and Nuclear Medicine, SLK Kliniken Heilbronn GmbH, Heilbronn, Germany
}

\begin{abstract}
Background and Aims: Intermediate stage hepatocellular carcinoma (HCC) can be treated by transarterial chemoembolization (TACE). However, there appear to be side effects, such as induction of proangiogenic factors, e.g. vascular endothelial growth factor (VEGF), which have been shown to be associated with a poor prognosis. This prospective study was designed to compare serum VEGF level response after TACE with different embolic agents in patients with HCC. Methods: Patients were assigned to one of three different TACE regimens: degradable starch microspheres (DSM) TACE, drugeluting bead (DEBDOX) TACE or Lipiodol TACE (CTACE). All patients received $50 \mathrm{mg}$ doxorubicin $/ \mathrm{m}^{2}$ body surface area (BSA) during TACE. Serum VEGF levels were assessed before TACE treatment, $24 \mathrm{~h}$ post-treatment and 4 weeks later. Results: Twenty-two patients with 30 TACE treatments were enrolled. Compared to baseline VEGF levels, a marked increase was observed for $24 \mathrm{~h}$ post-TACE ( $164 \%$ of baseline level) and during the 4-week follow-up (170\% of baseline level) only for the CTACE arm $(p<0.05)$. In contrast, the increase of serum VEGF levels were only $114 \%$ and $123 \%$ for DEBDOX and $121 \%$ and $124 \%$ for DSM, respectively. Conclusions: Conventional TACE using Lipiodol shows marked increase in blood levels of the proangiogenic factor VEGF, while DEBDOX and DSM TACE induce only a moderate VEGF response.

(C) 2016 The Second Affiliated Hospital of Chongqing Medical University. Published by XIA \& HE Publishing Inc. All rights reserved.
\end{abstract}

Keywords: Transarterial chemoembolization; Vascular endothelial growth factor; Embolic agents; Hepatocellular carcinoma; Angiogenesis.

Abbreviations: BSA, body surface area; BCLC, Barcelona Clinic Liver Cancer: CTACE, conventional TACE using Lipiodol; DEBDOX, drug eluting beads (with doxorubicin); DSM, degradable starch microspheres; HCC, hepatocellular carcinoma NO, nitric oxide; TACE, transarterial chemoembolization; VEGF, vascular endothelial growth factor.

Received: 04 November 2016; Revised: 05 December 2016; Accepted: 22 December 2016

DOI: $10.14218 /$ JCTH.2016.00058.

*Correspondence to: Andreas Schicho, Department of Radiology, University Hospital Regensburg, Franz-Josef-Strauß-Allee 11, Regensburg D-93053, Germany. Tel: +49-941-944-7412, Fax: +49-941-944-7469, E-mail: andreas. schicho@ukr.de

\section{Introduction}

Hepatocellular carcinoma (HCC) is one of the most frequent cancers worldwide. Treatment options are sparse and are highly dependent on the hepatic tumor infiltration. In the treatment of intermediate HCC Barcelona Clinic Liver Cancer (BCLC) stage $B$, conventional transarterial chemoembolization (CTACE) is an accepted and widely used treatment approach. This technique yields objective tumor response rates of approximately $60 \%-70 \%{ }^{1,2}$ and, when compared to best supportive care, a $20 \%-25 \%$ benefit of intermediateto long-term survival. ${ }^{3,4}$ Beyond the different treatment regimes, Lipiodol is found to be the predominant embolization compound, ${ }^{5}$ followed by drug-eluting beads $(D E B)^{6}$ and degradable starch microspheres (DSM), ${ }^{7}$ all in conjunction with doxorubicin, for example, as a DNA-intercalating cytotoxic agent. TACE thus affects the tumor in parallel, by delivering doxorubicin in a high concentration to the targeted tissue and reducing blood supply by embolization, thus leading to ischemia. ${ }^{8}$ As hypoxia is a definite threat to viable tissue, it affects a vast majority of intra- and intercellular signaling processes counteracting and reversing hypoxia. ${ }^{9}$ In malignant tissues, these biological countermeasures can potentially interfere with the underlying intention of antitumor treatment.

DSM-TACE, DEB-TACE, and CTACE show different embolization characteristics, which are crucial in the understanding of their mechanisms of biological action. While the beads used in DEB-TACE lead to an irreversible permanent embolization and ischemia, ${ }^{10}$ DSM-TACE leads to a transient ischemia with an occlusion half time of 35-50 minutes in vitro and 90-120 minutes in vivo in $\mathrm{HCC}$ patients (EmboCept $\left.{ }^{\circledR} \mathrm{S}\right) .{ }^{11,12}$ Lipiodol, the predominant chemoembolization agent, used in CTACE procedures has an ill-defined wide range of occlusion half time, ranging between 4 to 12 weeks. ${ }^{5}$

One of the key agents strongly activated by hypoxia, the vascular endothelial growth factor (VEGF), mediates angiogenesis and is thought to play a key role in tumor growth and metastatic seeding. ${ }^{13-15}$ Consequently, anti-VEGF therapies are being investigated as potential anti-cancer treatments and supportives. In $\mathrm{HCC}$, it was shown that a temporary overproduction of VEGF is caused by a single session of TACE; an increase of serum VEGF is related with future distant metastases, mainly in lungs and bones. ${ }^{16-18}$ Moreover, the postTACE peak of serum VEGF is an independent prognostic 
factor of progression-free survival in HCC. ${ }^{15,19}$ Anti-VEGF therapies have been reported and established in metastatic colorectal carcinoma in combination with other chemotherapeutic agents, ${ }^{20}$ macula degeneration ${ }^{21}$ and diabetic retinopathy. ${ }^{22}$ Consequently, avoidance of the post-TACE VEGF overproduction is of major interest for the treatment regimens of HCC patients, directly affecting their expected life span in a palliative situation.

Our prospective pilot-study addressed the hypothesis that the prolonged or permanent occlusion, other than the transient occlusion of DSM-TACE, causes a major and sustained VEGF response, possibly in accordance with the concept of an ischemia/reperfusion mechanism.

\section{Methods}

This two-center investigator-initiated pilot-study was approved by the local institutional review board. We included patients with intermediate stage HCC (BCLC stage B), either proven non-invasive by two imaging modalities or histologically. Subjects aged over 18 , presenting with 4 or more HCC nodules $\leq 3 \mathrm{~cm}$ or with a single lesion $\geq 3 \mathrm{~cm}$, and without portal vein invasion who had no contraindication for TACE were recruited for the study. We only included TACE-naïve patients; in cases of prior surgical resection or local ablation, at least 4 weeks had to pass before inclusion in the study.

Our study was designed to include 12 patients per treatment arm by randomization, addressing 3 different treatments; DEB-TACE (DEBDOX ${ }^{\mathrm{TM}}$; BTG International Ltd., London, UK), DSM-TACE (EmboCept ${ }^{\circledR}$ S; PharmaCept GmbH, Berlin, Germany), and CTACE (Lipiodol ${ }^{\circledR}$; Guerbet LLC, Bloomington, IN, USA). Dose of doxorubicin was $50 \mathrm{mg} / \mathrm{m}^{2}$ body surface area in each treatment arm. All patients included received standard TACE treatment according to a standard operating procedure as previously reported. ${ }^{23}$ Of 36 patients intended to include, 14 were either lost to 4 -weeks of follow-up $(n=7)$, samples having thawed on transport $(n=4)$, or relevant patient data missing $(n=3)$.

Plasma levels of VEGF were assessed right before transarterial therapy as baseline, $24 \mathrm{~h}$ after and 4 weeks after arterial treatment. A period of at least 4 weeks without intraarterial therapy was observed before further TACE. Peripheral whole blood samples were acquired according to our standard operating procedure, centrifuged at $1500 \mathrm{rpm}$ for 5 minutes and plasma samples were stored at $-20^{\circ} \mathrm{C}$ until shipping to a central laboratory on dry ice. VEGF levels in our samples were measured using a commercial ELISA kit (Human VEGF Quantikine ELISA Kit; R\&D Systems Inc., Minneapolis, MN, USA) by a third-party member not involved in patient inclusion, treatment or analysis of results. Plasma levels of VEGF right before TACE were set as reference (100\%).

Patient characteristics were analyzed by using descriptive statistics. A $t$-test or a Mann-Whitney U-test was used to compare metric data. A $p$-value $<0.05$ was considered to indicate a statistically significant difference. Statistical analyses were performed by using the JMP statistics software package (SAS Institute, Cary, NC, USA).

\section{Results}

\section{Patients characteristics}

In our pilot-study, complete data sets of 22 patients were obtained, represented by 9 for DSM-TACE, 7 for DEB-TACE,
Table 1. Baseline characteristics of patients and tumor burden

\begin{tabular}{llll}
\hline & DSM-TACE & DEB-TACE & cTACE \\
\hline $\begin{array}{l}\text { No. of patients } \\
\begin{array}{l}\text { No. of TACE- } \\
\text { cycles }\end{array}\end{array}$ & 9 & 7 & 6 \\
$\begin{array}{l}\text { Age, } \\
\text { mean } \pm \text { SD }\end{array}$ & $67 \pm 7.3$ & $70.9 \pm 7.7$ & $64.5 \pm 12.7$ \\
$\begin{array}{l}\text { Tumor burden, } \\
\text { cm } \pm \text { SD }\end{array}$ & $6.1 \pm 4.9$ & $5.2 \pm 4.3$ & $6.3 \pm 5.1$ \\
\hline
\end{tabular}

and 6 for CTACE (Table 1). Thereby, 9 TACE cycles were completed for DSM-TACE, 9 for DEB-TACE, and 12 for CTACE. Patients who received DSM-TACE were mean age of $67 \pm 7.3$ years, those who received DEB-TACE were $70.9 \pm 7.7$ years, and those who received CTACE were $64.5 \pm 12.7$; there were no statistically significant differences between the groups. Tumor burden was calculated as $6.1 \pm 4.9 \mathrm{~cm}$ (DSM-TACE), $5.2 \pm 4.3 \mathrm{~cm}$ (DEB-TACE), and $6.3 \pm 5.1 \mathrm{~cm}$ (CTACE), with no statistically significant differences between the groups.

\section{VEGF levels before and after different TACE procedures}

Plasma levels of VEGF right before TACE were set as reference $(100 \%)$. For DSM-TACE, plasma VEGF levels $24 \mathrm{~h}$ after TACE and 4 weeks later were elevated to $121 \pm 29 \%$ and $124 \pm$ $43 \%$, respectively. Results for DEB-TACE did not differ significantly, with $114 \pm 31 \%$ at $24 \mathrm{~h}$ after TACE ( $p=0.35$; Figs. 1 and 3 ) and $123 \pm 55 \%$ at 4 weeks later (Fig. 2).

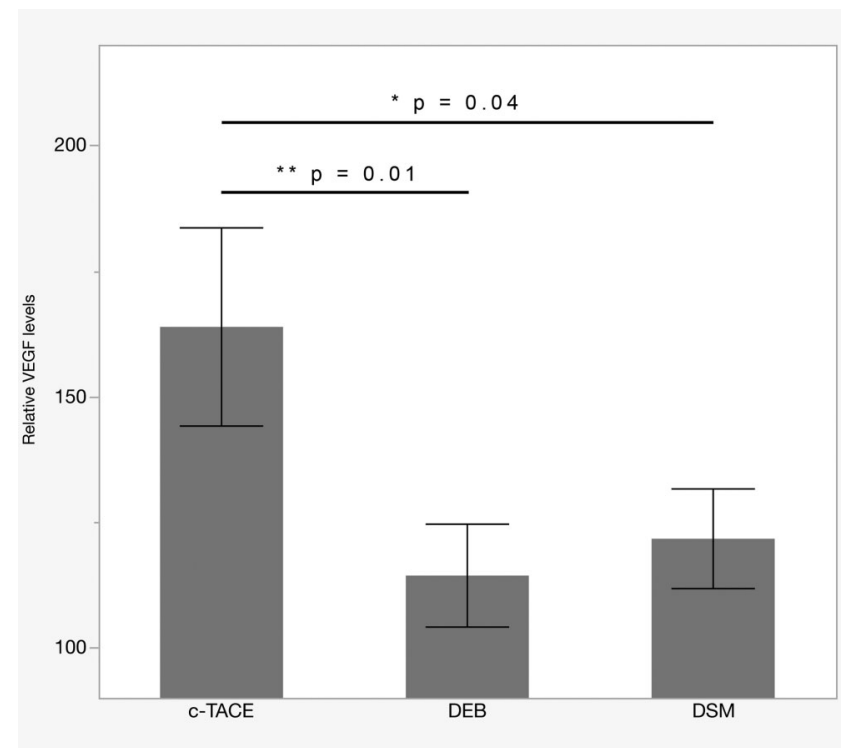

Fig. 1. Serum VEGF levels at $24 \mathrm{~h}$ after cTACE with standard Lipiodol, DEB, or DSM. Levels in DEB-TACE $(n=7)$ and DSM-TACE $(n=9)$ were significantly lower than in CTACE ( $n=6 ; p=0.01$ and $p=0.04$, respectively). Bars show mean \pm SEM.

Abbreviations: CTACE, conventional transarterial chemoembolization; DEB, drug eluting beads; DSM, degradable starch microspheres; VEGF, vascular endothelial growth factor. 
Schicho A. et al: Impact of TACE embolic agents on serum VEGF levels

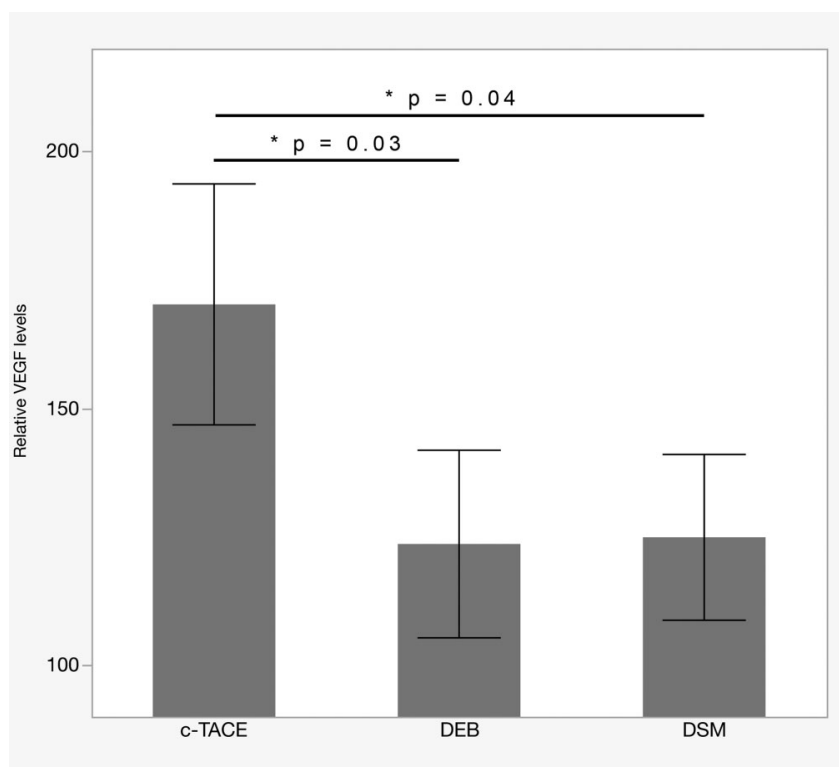

Fig. 2. Serum VEGF levels 1 month after cTACE with standard Lipiodol, DEB, or DSM. Levels in DEB-TACE $(n=7)$ and DSM-TACE $(n=9)$ were significantly lower than in CTACE $(n=6 ; p=0.03$ and $p=0.04$, respectively). Bars show mean \pm SEM.

Abbreviations: CTACE, conventional transarterial chemoembolization; DEB, drug eluting beads; DSM, degradable starch microspheres; VEGF, vascular endothelial growth factor.

In the CTACE group, VEGF plasma levels were elevated to $164 \pm 68 \%$ of baseline at $24 \mathrm{~h}$ post-TACE (vs. DSM-TACE: $p=0.04$ and vs. DEB-TACE: $p=0.01 ;$ Fig. 1 ) and remained at high levels $(170 \pm 81 \%)$ for at least 4 weeks after the treatment (vs. DSM-TACE: $p=0.04$ and vs. DEB-TACE: $p=0.03$; Fig. 2).

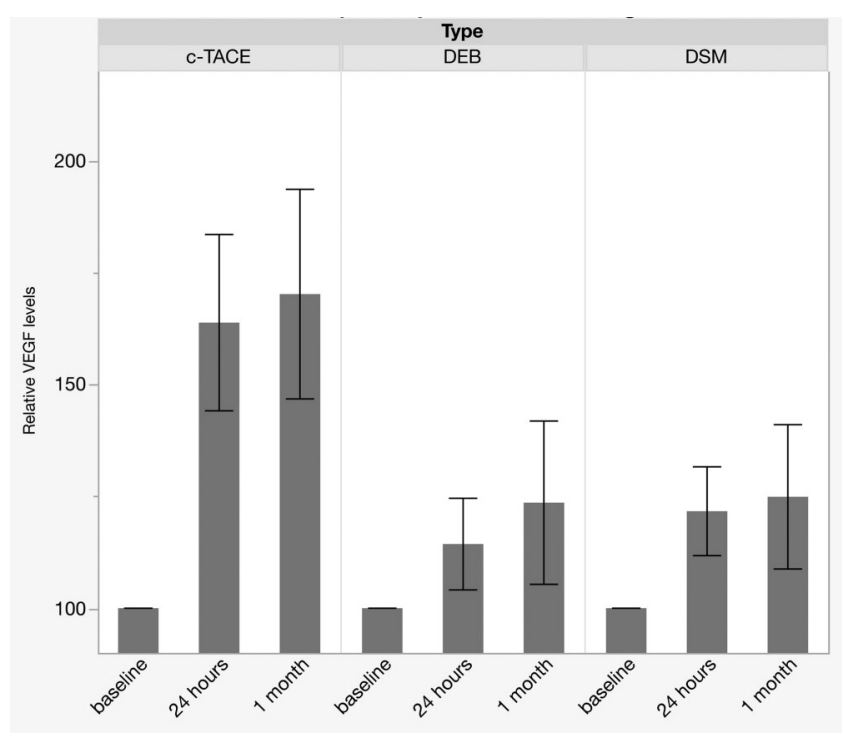

Fig. 3. Serum-VEGF levels at $24 \mathrm{~h}$ and 1 month post-treatment compared to baseline. Bars show mean \pm SEM.

Abbreviation: VEGF, vascular endothelial growth factor.

\section{Discussion}

VEGF plays a key role in hypoxia signaling. ${ }^{14}$ Elevated levels are found in a vast variety of tissues when put at risk of tissue-threatening ischemia, e.g. in heart (myocardial infarction), ${ }^{24}$ brain (stroke), ${ }^{25}$ muscle (peripheral arterial occlusive disease), ${ }^{26}$ liver (tumor, infarction) ${ }^{27}$ and kidney (infarction). ${ }^{28}$ While VEGF causes both long- and short-term tissue protective effects in healthy tissue, its effects in tumorous tissues include, but are not limited to, faster and more profound metastatic seeding, as well as regeneration and growth of malignant tissues. ${ }^{16-18}$

Since its first publication in the late 1980 's, ${ }^{29}$ CTACE became a standard treatment in intermediate stage HCC. ${ }^{30}$ In the meantime, other technologies have been reported, including $\mathrm{DSM}^{7}{ }^{7} \mathrm{DEB},{ }^{6}$ and Lipiodol as the so-called 'conventional' CTACE. $^{5}$ Among the factors potentially interfering with its effectiveness is a potent VEGF-dependent neoangiogenic response due to the ischemia (and reperfusion) caused by the embolization agents, which shows characteristic occlusion half-life values of 90-120 minutes for DSM-TACE, 4 to 12 weeks in Lipiodol CTACE, and irreversible occlusion in DEBTACE. Therefore, we set up a pilot-study to assess changes in VEGF plasma levels depending on the used embolic agents.

Our results show a rise of the VEGF plasma levels above baseline in all three treatments, as a result of ischemia caused by embolization. While the permanent embolization by DEB-TACE and the transient ischemia in DSM-TACE yielded levels of $7 \%$ and $22 \%$ above baseline at $24 \mathrm{~h}$, respectively, the persistent but non-permanent embolization in CTACE causes a significantly enlarged (1.7-fold) rise in VEGF plasma levels. These elevations essentially remained unchanged for (at least) 4 weeks following the TACE procedure in all three studied substances. Thus, Lipiodol cTACE patients are subject to a profound VEGF release for at least 4 weeks. These post-cTACE results are in line with prior published results of both human and animal study protocols; Sergio et al. ${ }^{19}$ even found a further increase of VEGF in their follow-up of 4 weeks after CTACE. According to the data of 8 patients receiving two TACE treatments in our study, an accumulative effect seems possible; further studies are needed to clarify the VEGF serum kinetics after TACE.

\section{Potential underlying mechanism}

A hypothetic key to understanding the significant changes arising from the different chemoembolization agents are the chemical properties of the oily Lipiodol emulsion, causing an "unstable" ischemia with reperfusion situation in the targeted tissue. While, to the contrary, DSM has a defined occlusion time of 90 to 120 minutes in vivo, and Lipiodol has an occlusion half time ranging between 4 and 12 weeks. It is known that sustained ischemia either causes tissue death by both necrosis and apoptosis, ${ }^{26,31,32}$ or in case of survival, regeneration and adaptation of the tissue. Other than Lipiodol, DEB-TACE does not enable a reperfusion of the embolized, ischemic tissue, which is crucial for the cellular and humoral response to hypoxia. DSM-TACE on the other hand seems to not yield a durable tissue ischemia, long enough to elicit a stronger response in VEGF plasma levels. As an oily emulsion, Lipiodol has no defined particle size, enabling a sustained but dynamic situation of tissue hypoxia with subsequent, perpetual reperfusion. 
From a completely different set of organs, ${ }^{33,34}$ as well as liver, $^{35}$ it is known that reperfusion is an essential component in eliciting both strong cellular and humoral signaling processes, including the expression of cytokines, growth factors and adhesion molecules that are repeatedly reported to foster growth of malignant tumors. Microcirculatory dysfunction, as assumed to be predominant in CTACE, has repeatedly been found to resemble a source of hypoxia. ${ }^{36}$ Mechanistically, besides hypoxia-inducible factor (HIF-) $1 a,{ }^{37}$ which acts as a cell survival factor, and Fas-Fas ligand interactions, ${ }^{38}$ essential in the regulation of both apoptosis and necrosis, the main component reportedly is the up-regulation of VEGF expression.

VEGF causes tumor angiogenesis, and it was shown that, especially in the liver, ischemia/reperfusion-triggered up-regulation of VEGF causes improved tumor vascularization. Moreover, positive-feedback autoregulatory functions of VEGF were found via a parallel overexpression of VEGF receptors in malignant cells, including HCC cells. Recent reports additionally credit side effects like promotion of cancer cell survival and cancer cell migration to the up-regulation of VEGF and the VEGF receptor. ${ }^{39}$

\section{Clinical implication}

When transferring these reported observations to the clinical setting of HCC treatment by TACE, avoiding the use of Lipiodol in favor of DEB- or DSM-TACE could directly affect the clinical outcome of HCC patients; it was shown that the temporary overexpression of circulating VEGF caused by a single session of CTACE can contribute to inducing future distant metastatic seeding, mainly pulmonary and osseous. ${ }^{18}$ The VEGF serum levels, moreover, were shown to be an independent prognostic factor of progression-free survival in HCC treated with cTACE. Therefore, it should be of major interest to preserve low VEGF levels in HCC patients. Prevention of ischemia-induced microcirculation with L-arginine (an enhancer of endothelial nitric oxide synthesis) reduces the seeding of micrometastases by minimizing tissue hypoxia, and direct scavenging of free-circulating VEGF by the antiVEGF monoclonal antibody bevacizumab is approved by the FDA as first-line therapy, e.g. in metastatic colorectal carcinoma, in combination with other chemotherapeutic agents and, moreover, is used on- and off-label in a wide variety of oncology and non-oncology indications, including macular degeneration and diabetic retinopathy. ${ }^{22}$

While these approaches try to ameliorate an apparently TACE-inherent VEGF up-regulation, the results of our pilotstudy support the use of DEB- or DSM-TACE instead of Lipiodol as the first causally determined treatment of VEGF overexpression. Additionally, differences in safety and side effects of the embolic agents have to be observed and taken into account.

\section{Limitations}

Since our study was designed as a pilot-study in a proof-ofprinciple fashion, some inherent limitations have to be considered. The small sample size in each treatment arm may limit generalizability of results. Nonetheless, implications derived from our results for clinical treatment need to be tested in a larger cohort, e.g. investigation of potential differences between DEB-TACE and DSM-TACE, or safety and side effects of different embolic agents.
The major physiological transporters of VEGF in blood are circulating platelets. It is known that VEGF concentrations highly correlate with platelet counts. Moreover, the platelet load of VEGF in patients with malignant tumors correlates quantitatively with the expression of VEGF in malignant tissues. Our study design did not cover collection and analysis of platelet counts. Both platelet count changes as well as changes of VEGF receptor expression should be addressed by future studies.

Previous studies have shown that pre-TACE levels per se are correlated with HCC characteristics, including tumor size, vascular invasion and metastasis. Thus, further studies should allow for an in-depth analysis of baseline VEGF-levels in HCC patients.

\section{Conclusions}

Among the factors potentially interfering with the intention of TACE in HCC, post-interventional up-regulation of VEGF is of major concern, since VEGF is known to promote tumor growth via neoangiogenesis, metastatic seeding, and both cancer cell migration and survival. The widely used cTACE causes an enlarged VEGF serum response sustaining for at least 28 days post-TACE. On contrary, both DEB-TACE and DSM-TACE elicit a significantly lower VEGF response, making the preferred use of these chemoembolization agents the first causally determined approach to an ameliorated post-TACE VEGF overexpression. Parallel use of anti-VEGF therapies could be of value in the treatment of HCC.

\section{Acknowledgments}

Financial assistance from PharmaCept $\mathrm{GmbH}$, Berlin, Germany to Stroszczynski C and Wiggermann P.

\section{Conflict of interest}

None

\section{Author contributions}

Study design (CS, PLP, PW), patient inclusion and performance of transarterial chemoembolization (WW, EH, CS, PLP, PW), sample preparation and obtainment of patient data (AS, KK, LPB, CN), vascular endothelial growth factor serum level analysis $(\mathrm{CH})$, data analysis (AS, LPB, PW), manuscript drafting and figure preparation (AS, LPB), discussion and approval of manuscript (AS, CH, KK, LPB, WW, CN, EH, CS, PLP, PW), supervision (CS, PLP, PW).

\section{References}

[1] Hu HT, Kim JH, Lee LS, Kim KA, Ko GY, Yoon HK, et al. Chemoembolization for hepatocellular carcinoma: multivariate analysis of predicting factors for tumor response and survival in a 362-patient cohort. J Vasc Interv Radiol 2011;22:917-923. doi: 10.1016/j.jvir.2011.03.005.

[2] Lewandowski RJ, Mulcahy MF, Kulik LM, Riaz A, Ryu RK, Baker TB, et al. Chemoembolization for hepatocellular carcinoma: comprehensive imaging and survival analysis in a 172-patient cohort. Radiology 2010;255:955965. doi: 10.1148/radiol.10091473.

[3] Llovet JM, Real MI, Montaña X, Planas R, Coll S, Aponte J, et al. Arterial embolisation or chemoembolisation versus symptomatic treatment in patients with unresectable hepatocellular carcinoma: a randomised controlled trial. Lancet 2002;359:1734-1739. doi: 10.1016/S0140-6736(02) 08649-X. 
[4] Lo CM, Ngan H, Tso WK, Liu CL, Lam CM, Poon RT, et al. Randomized controlled trial of transarterial lipiodol chemoembolization for unresectable hepatocellular carcinoma. Hepatology 2002;35:1164-1171. doi: 10.1053/jhep. 2002.33156.

[5] Lencioni R, de Baere T, Soulen MC, Rilling WS, Geschwind JF. Lipiodol transarterial chemoembolization for hepatocellular carcinoma: A systematic review of efficacy and safety data. Hepatology 2016;64:106-116. doi: 10. 1002/hep.28453.

[6] Lewis AL, Dreher MR. Locoregional drug delivery using image-guided intraarterial drug eluting bead therapy. J Control Release 2012;161:338-350. doi: 10.1016/j.jconrel.2012.01.018.

[7] Orlacchio A, Chegai F, Merolla S, Francioso S, Giudice $C D$, Angelico $M$, et al. Downstaging disease in patients with hepatocellular carcinoma outside upto-seven criteria: Strategies using degradable starch microspheres transcatheter arterial chemo-embolization. World J Hepatol 2015;7:1694-1700. doi: 10.4254 /wjh.v7.i12.1694.

[8] Choi J. Regional transcatheter therapy of hepatic neoplasms. Cancer Control 1996;3:407-413.

[9] Carmeliet P. Angiogenesis in life, disease and medicine. Nature 2005;438: 932-936. doi: 10.1038/nature04478.

[10] Facciorusso A, Di Maso M, Muscatiello N. Drug-eluting beads versus conventional chemoembolization for the treatment of unresectable hepatocellular carcinoma: A meta-analysis. Dig Liver Dis 2016;48:571-577. doi: 10.1016/ j.dld.2016.02.005.

[11] Ebert M, Ebert J, Berger G. Intravital microscopic research of microembolization with degradable starch microspheres. J Drug Deliv 2013;2013: 242060. doi: 10.1155/2013/242060.

[12] Wiggermann P, Wohlgemuth WA, Heibl M, Vasilj A, Loss M, Schreyer AG, et al. Dynamic evaluation and quantification of microvascularization during degradable starch microspheres transarterial Chemoembolisation (DSMTACE) of HCC lesions using contrast enhanced ultrasound (CEUS): a feasibility study. Clin Hemorheol Microcirc 2013;53:337-348. doi: $10.3233 / \mathrm{CH}-$ 2012-1555.

[13] Miura H, Miyazaki T, Kuroda M, Oka T, Machinami R, Kodama T, et al. Increased expression of vascular endothelial growth factor in human hepatocellular carcinoma. J Hepatol 1997;27:854-861.

[14] Semela D, Dufour JF. Vascular endothelial growth factor signaling. Heidelberg: Springer-Verlag Berlin, 2005.

[15] Yamaguchi R, Yano $H$, Iemura A, Ogasawara S, Haramaki M, Kojiro M. Expression of vascular endothelial growth factor in human hepatocellular carcinoma. Hepatology 1998;28:68-77. doi: 10.1002/hep.510280111.

[16] Li X, Feng GS, Zheng CS, Zhuo CK, Liu X. Expression of plasma vascular endothelial growth factor in patients with hepatocellular carcinoma and effect of transcatheter arterial chemoembolization therapy on plasma vascular endothelial growth factor level. World J Gastroenterol 2004;10: 2878-2882.

[17] Poon RT, Lau C, Yu WC, Fan ST, Wong J. High serum levels of vascular endothelial growth factor predict poor response to transarterial chemoembolization in hepatocellular carcinoma: a prospective study. Oncol Rep 2004;11: $1077-1084$.

[18] Xiong ZP, Yang SR, Liang ZY, Xiao EH, Yu XP, Zhou SK, et al. Association between vascular endothelial growth factor and metastasis after transcatheter arterial chemoembolization in patients with hepatocellular carcinoma. Hepatobiliary Pancreat Dis Int 2004;3:386-390.

[19] Sergio A, Cristofori C, Cardin R, Pivetta G, Ragazzi R, Baldan A, et al. Transcatheter arterial chemoembolization (TACE) in hepatocellular carcinoma (HCC): the role of angiogenesis and invasiveness. Am J Gastroenterol 2008;103:914-921. doi: 10.1111/j.1572-0241.2007.01712.x.

[20] Tampellini M, Sonetto C, Scagliotti GV. Novel anti-angiogenic therapeutic strategies in colorectal cancer. Expert Opin Investig Drugs 2016;25: 507-520. doi: 10.1517/13543784.2016.1161754.

[21] Arevalo JF, Lasave AF, Wu L, Acon D, Farah ME, Gallego-Pinazo R, et al. Intravitreal bevacizumab for diabetic macular oedema: 5-year results of the
Pan-American Collaborative Retina Study group. Br J Ophthalmol 2016; 100:1605-1610. doi: 10.1136/bjophthalmol-2015-307950.

[22] Wells JA, Glassman AR, Ayala AR, Jampol LM, Bressler NM, Bressler SB, et al. Aflibercept, bevacizumab, or ranibizumab for diabetic macular edema: twoyear results from a comparative effectiveness randomized clinical trial. Ophthalmology 2016;123:1351-1359. doi: 10.1016/j.ophtha.2016.02.022.

[23] Wiggermann P, Sieron D, Brosche C, Brauer T, Scheer F, Platzek I, et al. Transarterial chemoembolization of child-a hepatocellular carcinoma: drugeluting bead TACE (DEB TACE) vs. TACE with cisplatin/lipiodol (CTACE). Med Sci Monit 2011;17:CR189-195. doi: 10.12659/MSM.881714.

[24] Cheng C, Li P, Wang YG, Bi MH, Wu PS. Study on the expression of VEGF and HIF-1 $\alpha$ in infarct area of rats with AMI. Eur Rev Med Pharmacol Sci 2016;20: $115-119$.

[25] Jiang C, Zuo F, Wang Y, Lu H, Yang Q, Wang J. Progesterone Changes VEGF and BDNF Expression and Promotes Neurogenesis After Ischemic Stroke. Mol Neurobiol 2016. doi: 10.1007/s12035-015-9651-y.

[26] Hendgen-Cotta UB, Luedike P, Totzeck M, Kropp M, Schicho A, Stock P, et al. Dietary nitrate supplementation improves revascularization in chronic ischemia. Circulation 2012;126:1983-1992. doi: 10.1161/CIRCULATIONAHA. 112.112912.

[27] Hato T, Zhu AX, Duda DG. Rationally combining anti-VEGF therapy with checkpoint inhibitors in hepatocellular carcinoma. Immunotherapy $2016 ; 8$ : 299-313. doi: 10.2217/imt.15.126.

[28] Gnudi L, Benedetti S, Woolf AS, Long DA. Vascular growth factors play critical roles in kidney glomeruli. Clin Sci (Lond) 2015;129:1225-1236. doi: 10. $1042 / C S 20150403$.

[29] Hidaka H, Kobayashi H, Ohyama M, Maeda T, Ikeda K, Nakajo M, et al. Transarterial chemoembolization therapy of hepatocellular carcinoma using anticancer agents (mitomycin C and/or adriamycin) suspended in lipiodol. Nihon Igaku Hoshasen Gakkai Zasshi 1985;45:1430-1440.

[30] Llovet JM, Bruix J. Systematic review of randomized trials for unresectable hepatocellular carcinoma: Chemoembolization improves survival. Hepatology 2003;37:429-442. doi: 10.1053/jhep.2003.50047.

[31] Brenner C, Galluzzi L, Kepp O, Kroemer G. Decoding cell death signals in liver inflammation. J Hepatol 2013;59:583-594. doi: 10.1016/j.jhep.2013. 03.033.

[32] Totzeck M, Schicho A, Stock P, Kelm M, Rassaf T, Hendgen-Cotta UB. Nitrite circumvents canonical cGMP signaling to enhance proliferation of myocyte precursor cells. Mol Cell Biochem 2015;401:175-183. doi: 10.1007/ s11010-014-2305-y.

[33] Totzeck M, Hendgen-Cotta U, Rassaf T. Concepts of hypoxic NO signaling in remote ischemic preconditioning. World J Cardiol 2015;7:645-651. doi: 10. 4330/wjc.v7.i10.645

[34] Totzeck M, Hendgen-Cotta UB, Luedike P, Berenbrink M, Klare JP, Steinhoff $\mathrm{HJ}$, et al. Nitrite regulates hypoxic vasodilation via myoglobin-dependent nitric oxide generation. Circulation 2012;126:325-334. doi: 10.1161/ CIRCULATIONAHA.111.087155.

[35] Siniscalchi A, Gamberini L, Laici C, Bardi T, Ercolani G, Lorenzini L, et al. Post reperfusion syndrome during liver transplantation: From pathophysiology to therapy and preventive strategies. World J Gastroenterol 2016;22: 1551-1569. doi: 10.3748/wjg.v22.i4.1551.

[36] Weigand K, Brost S, Steinebrunner N, Büchler M, Schemmer P, Müller M. Ischemia/Reperfusion injury in liver surgery and transplantation: pathophysiology. HPB Surg 2012;2012:176723. doi: 10.1155/2012/176723.

[37] Span PN, Bussink J. Biology of hypoxia. Semin Nucl Med 2015;45:101-109. doi: $10.1053 /$ j.semnuclmed.2014.10.002.

[38] Nagata S, Golstein P. The Fas death factor. Science 1995;267:1449-1456.

[39] Wang F, He Z, Dai W, Li Q, Liu X, Zhang Z, et al. The role of the vascular endothelial growth factor/vascular endothelial growth factor receptors axis mediated angiogenesis in curcumin-loaded nanostructured lipid carriers induced human HepG2 cells apoptosis. J Cancer Res Ther 2015;11:597-605. doi: $10.4103 / 0973-1482.159086$. 